\title{
Antiphospholipid syndrome in lupus retinopathy
}

This article was published in the following Dove Press journal:

Clinical Ophthalmology

24 November 2014

Number of times this article has been viewed

\author{
$\mathrm{Ng}$ Hong-Kee ${ }^{1,2}$ \\ Chong Mei-Fong ${ }^{2}$ \\ Yaakub Azhany' \\ Embong Zunaina' \\ 'Department of Ophthalmology, \\ School of Medical Sciences, Universiti \\ Sains Malaysia, Kubang Kerian, \\ Kelantan, Malaysia; ${ }^{2}$ Department \\ of Ophthalmology, Hospital Raja \\ Permaisuri Bainun, Ipoh, Perak, \\ Malaysia
}

\begin{abstract}
Systemic lupus erythematosus (SLE) is a chronic, multisystem, autoimmune disease that can affect any part of the human body including the eyes. Common blinding ocular manifestations include central retinal artery occlusion (CRAO), central retinal vein occlusion (CRVO), severe vaso-occlusive retinopathy, and optic nerve involvement. Antiphospholipid syndrome (APS) in lupus is usually associated with large vessel occlusions and needs prompt treatment with anticoagulant. We are reporting two cases of APS in SLE patients that presented with CRVO (case 1) and vaso-occlusive lupus retinopathy (case 2). Both cases were positive for antiphospholipid antibody (APA) and were treated with immunosuppression, anticoagulant, and laser treatment. Thus, screening for APA is vital in SLE patients with lupus retinopathy, as prompt treatment with anticoagulants is important to prevent further vascular thrombosis, which worsens the visual prognosis.
\end{abstract}

Keywords: systemic lupus erythematosus, antiphospholipid antibody, central retinal vein occlusion, vaso-occlusive retinopathy

\section{Introduction}

Systemic lupus erythematosus (SLE) is a chronic, multisystem, autoimmune disease that can affect any part of the human body. Ocular manifestations in SLE are fairly common and should be taken seriously as they may lead to significant morbidity and visual deterioration. In addition to that, ocular manifestations could represent the disease activity of the lupus (especially in the presence of retinal signs) and serve as an indicator of adequacy of treatment in control of the disease. ${ }^{1}$ Common ocular signs that could lead to visual blinding complications include retinal involvement (central retinal artery occlusion [CRAO], central retinal vein occlusion [CRVO], severe vasoocclusive retinopathy, and retinal detachment) and optic nerve involvement (optic neuritis and ischemic optic neuropathy). ${ }^{2}$ Milder spectrum includes keratoconjunctivitis sicca, episcleritis, scleritis, and anterior uveitis.

Antiphospholipid antibodies (APA) have been recognized as a risk factor for complications in SLE and their clinical significance in ocular presentation have been associated with poorer prognosis. ${ }^{3}$ Clinicians will usually raise their suspicion of antiphospholipid syndrome (APS) in SLE patients if there is large vessel involvement such as CRAO or CRVO. We are reporting two cases of APS in SLE patients that presented with CRVO (case 1) and vaso-occlusive lupus retinopathy (case 2).

\section{Case series}

\section{Case I}

A 19-year-old girl with known SLE for 3 years and was on maintenance oral prednisolone $10 \mathrm{mg}$ daily presented in the ophthalmology clinic at the Hospital Raja Permaisuri Bainun, Ipoh, Malaysia with sudden, painless loss of vision in the left eye of 1 day's duration. There were no other manifestations of SLE or thrombotic events. Visual acuity was hand movement in the left eye with the presence of relative 


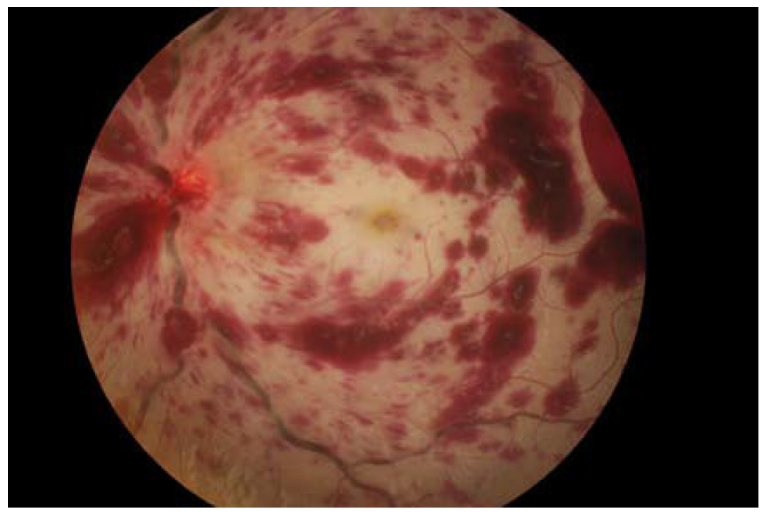

Figure I Left fundus showed extensive flame-shaped intraretinal hemorrhages, and dilated and tortuous veins with swollen and hyperemic disc at presentation.

afferent pupillary defect (RAPD), and was 6/6 in the right eye. Anterior segment examinations of both eyes were unremarkable. Left fundoscopy showed extensive flameshaped intraretinal hemorrhages, dilated and tortuous retinal veins with swollen and hyperemic disc suggestive of CRVO (Figure 1). Right fundoscopy was unremarkable. Fundus fluorescein angiography (FFA) of the left eye revealed delayed venous filling and prolonged arteriovenous transit time with extensive capillary nonperfusion affecting $360^{\circ}$ of peripheral retina with macular ischemia (Figure 2). FFA of the right eye was unremarkable.

In view of the ocular vascular thrombotic event, she was screened for laboratory criteria for APS, which consisted of lupus anticoagulant, anticardiolipin antibody, and $\beta-2$ glycoprotein-1 antibody. The screening revealed that the anticardiolipin antibody was positive at a moderate titer (IgG: 73.85 GPL units, IgM: 8.62 MPL units).

She was given intravenous methylprednisolone $500 \mathrm{mg}$ daily for 3 days followed by oral prednisolone $55 \mathrm{mg}$

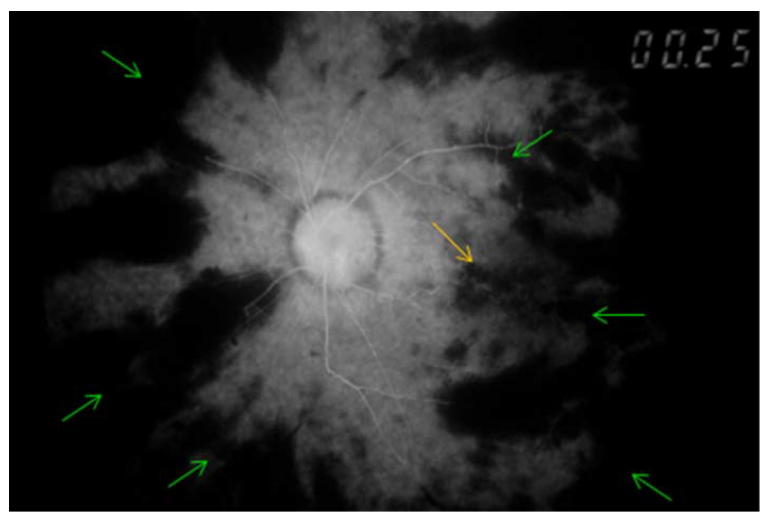

Figure 2 Fundus fluorescein angiography of left fundus showed extensive capillary nonperfusion affecting $360^{\circ}$ of peripheral retina (green arrows) with macular ischemia (yellow arrow).

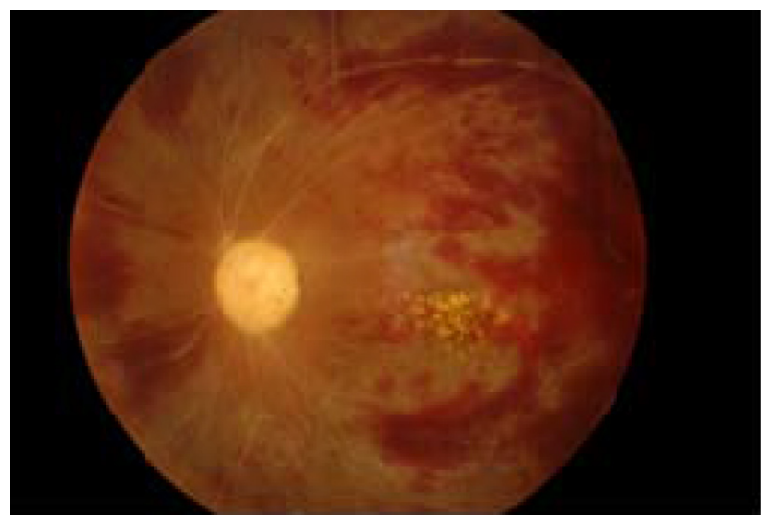

Figure 3 Left fundus showed pale optic disc with resolving retinal hemorrhages and extensive sclerosed retinal vessels at week 16 of follow-up.

( $1 \mathrm{mg} / \mathrm{kg}$ daily) for 2 weeks. Oral prednisolone was tapered down $10 \mathrm{mg}$ every 2 weeks until $30 \mathrm{mg}$, and then further tapered down $5 \mathrm{mg}$ weekly until maintenance of $5 \mathrm{mg}$ daily. The patient was also treated with oral hydroxychloroquine (HCQ) $200 \mathrm{mg}$ daily and oral warfarin $5 \mathrm{mg}$ daily. She was reviewed regularly for international normalization ratio (INR) aiming at 2.0-3.0. She was planned for long-term treatment of HCQ and oral anticoagulant. In view of the extensive capillary nonperfusion area in the left eye, laser panretinal photocoagulation (PRP) was given in three sessions with a total of 3,000 shots.

Follow-up review at 16 weeks revealed her left visual acuity was 6/60. The left fundus showed pallor disc with resolving retinal hemorrhages with extensive sclerosed retinal vessels (Figure 3). She is still on maintenance treatment with oral prednisolone $5 \mathrm{mg}$ daily, oral HCQ $200 \mathrm{mg}$ daily, and oral warfarin $5 \mathrm{mg}$ daily.

\section{Case 2}

A 16-year-old girl with known SLE and lupus nephritis for 10 months, on maintenance oral prednisolone $20 \mathrm{mg}$ daily, oral mycophenolate mofetil (MMF) $750 \mathrm{mg}$ daily, and oral HCQ $200 \mathrm{mg}$ twice daily. She had a history of deep vein thrombosis (DVT) 18 months ago. Her ultrasound Doppler showed thrombosis of the right external iliac and common femoral vein and was started on oral warfarin for 6 months duration. She presented to the ophthalmology clinic in Hospital Raja Permaisuri Bainun, Ipoh, Malaysia with bilateral central scotomas of 4 days' duration. The visual acuity was 6/24 bilaterally with no RAPD. Anterior segment examinations of both eyes were unremarkable. Fundoscopy showed multiple cotton wool spots, intraretinal hemorrhages, and macular edema bilaterally (Figure 4A and B). FFA showed perifoveal vasculitis with macular ischemia and capillary 

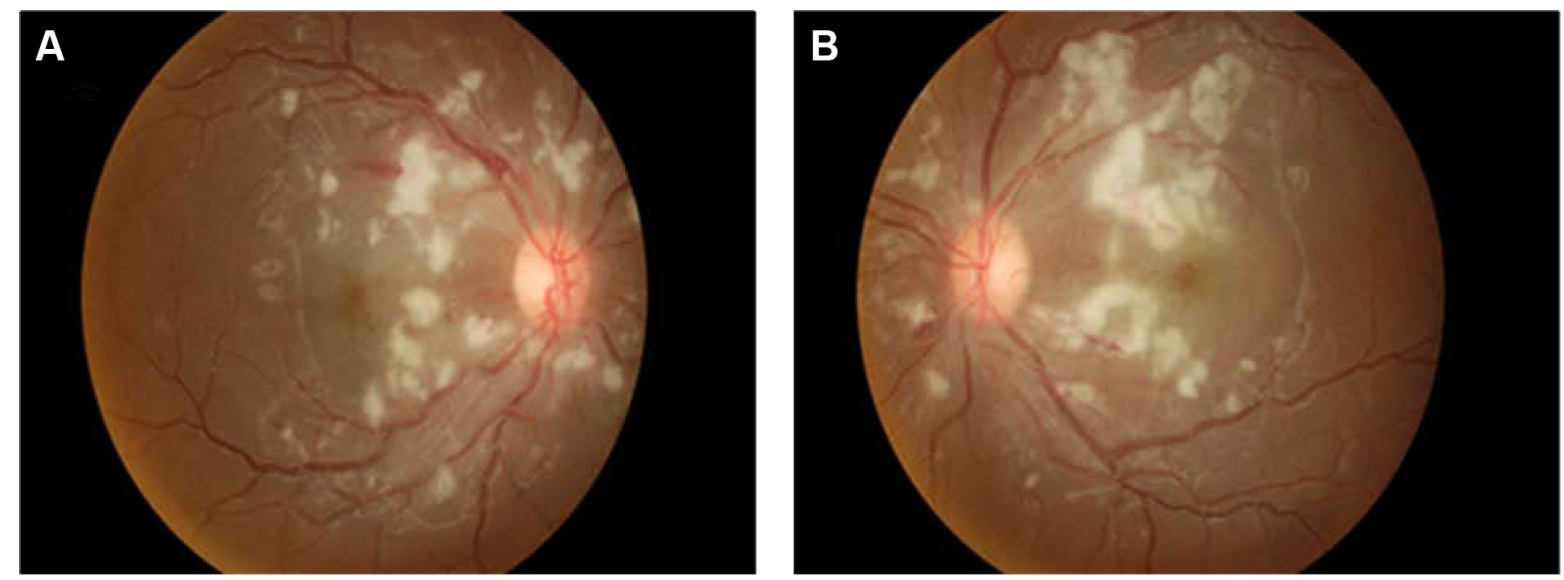

Figure 4 Fundus findings at presentation for both eyes in case 2.

Note: Both fundus showed multiple cotton wool spots and intraretinal hemorrhages at presentation (A [right eye] and B [left eye]).

nonperfusion at superior, temporal, and inferior parts of the retina in both eyes (the left eye more severe than the right eye) (Figure 5A and B). These FFA findings were in keeping with vaso-occlusive lupus retinopathy. APA screening showed positive anticardiolipin antibody (IgG:12.6 GPL units) and lupus anticoagulant (ratio between 1.2 to 1.5 ).

She was given intravenous methylprednisolone $500 \mathrm{mg}$ daily for 3 days followed by oral prednisolone $45 \mathrm{mg}$ daily $(1 \mathrm{mg} / \mathrm{kg}$ daily), then tapered down $10 \mathrm{mg}$ every 2 weeks until maintenance of $10 \mathrm{mg}$ daily. Oral anticoagulant (warfarin $5 \mathrm{mg}$ daily) was commenced. She was reviewed regularly for INR with the aim of 2.0 to 3.0. She was planned for long-term oral anticoagulant. In view of vaso-occlusive retinopathy at peripheral retina, laser PRP was given in both eyes at superior, temporal, and inferior parts of the retina in three sessions to prevent neovascularization.

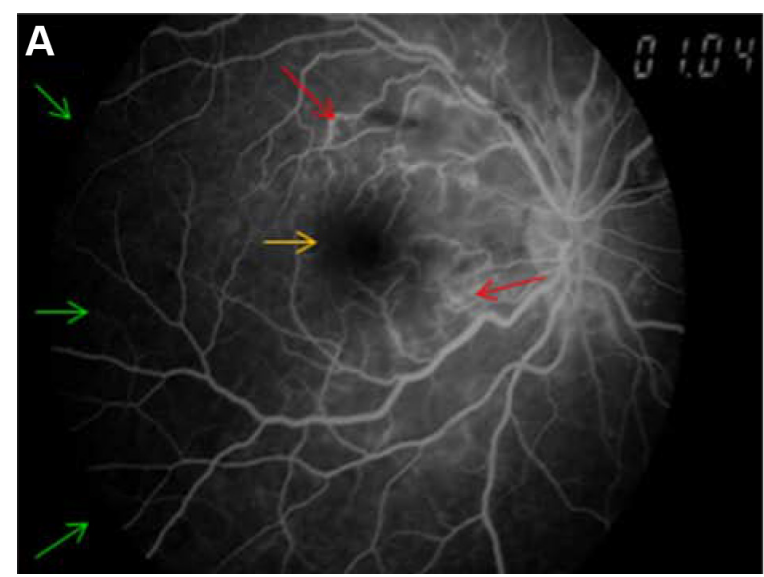

At 12 weeks follow-up, her visual acuity was 6/12 in the right eye and 6/24 in the left eye with persistent central scotomas bilaterally. Both fundus showed resolving of cotton wool spots and intraretinal hemorrhages with the presence of hard exudate at the macular area (Figure 6A and B). She is still on maintenance treatment with oral prednisolone $10 \mathrm{mg}$ daily, oral warfarin $5 \mathrm{mg}$ daily, oral MMF $750 \mathrm{mg}$ daily, and oral HCQ $200 \mathrm{mg}$ twice daily.

\section{Discussion}

APS is an autoimmune disorder that frequently affects young women and leads to recurrent venous or arterial thrombosis and/or fetal loss. Diagnosis of APS requires one out of two clinical criteria (vascular thrombosis or pregnancy morbidity) and one laboratory criteria that includes either lupus anticoagulant, anticardiolipin antibody in medium or high

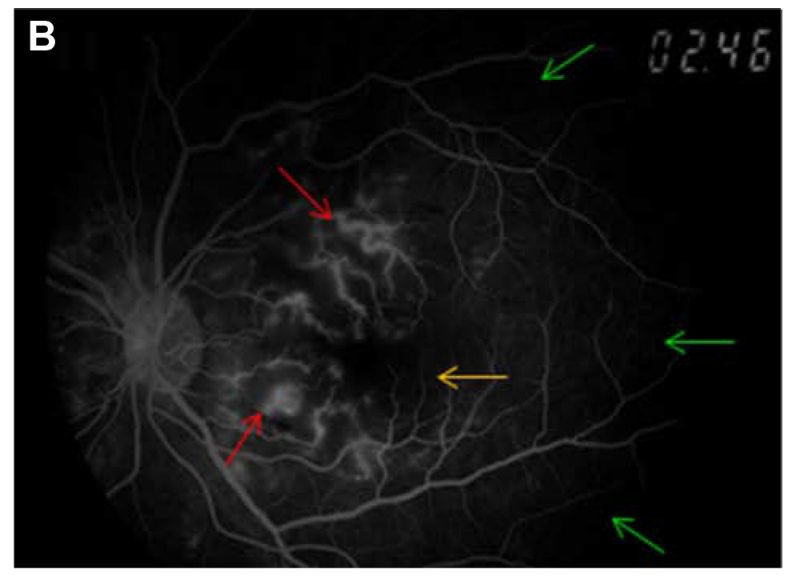

Figure 5 Fundus fluorescein angiography for both eyes in case 2 .

Note: Fundus fluorescein angiography showed perifoveal vasculitis (red arrows) with macular ischemia (yellow arrows) and capillary nonperfusion (green arrows) at superior, temporal, and inferior parts of the retina in both eyes ( $\mathbf{A}$ [right eye] and $\mathbf{B}$ [left eye]). 

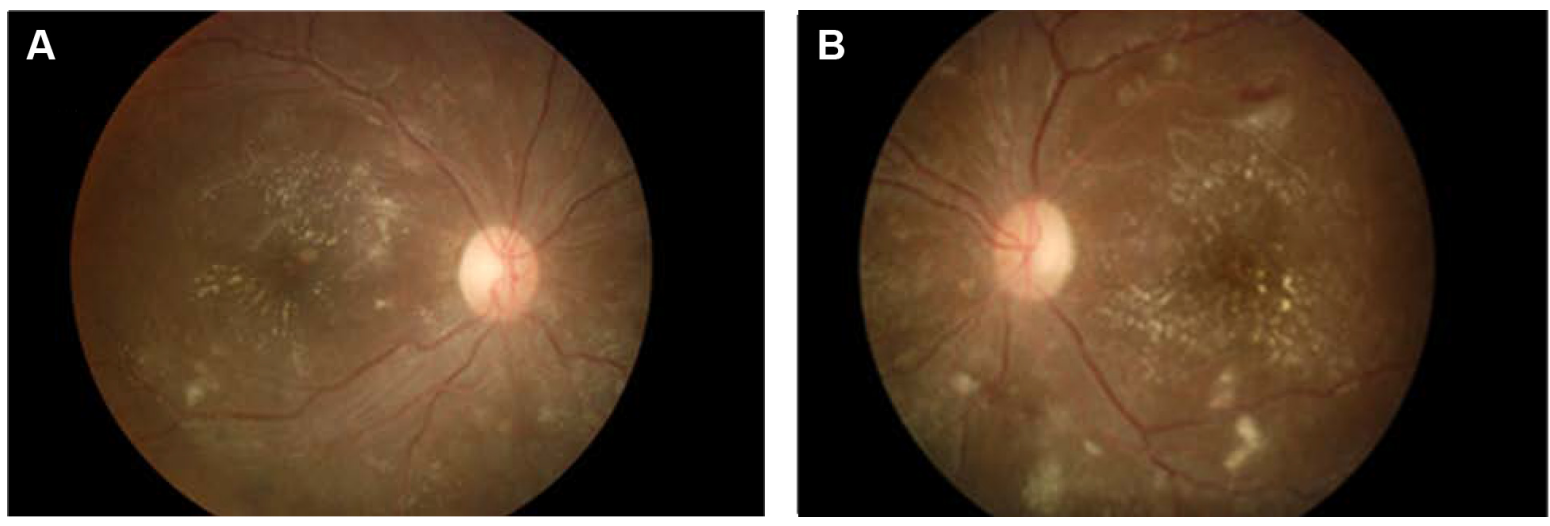

Figure 6 Fundus findings at week 12 follow-up for both eyes in case 2.

Note: Both fundus showed resolving of cotton wool spots and intraretinal hemorrhages with presence of hard exudates at macular area at week 12 of follow-up (A [right eye] and B [left eye]).

titer, or anti- $\beta 2$ glycoprotein-1 antibody. The presence of these autoantibodies causes imbalance to the hemostasis, which favors the prothrombotic state, thus leading to arterial and venous thrombosis, and is associated with more severe retinopathy and vascular occlusion. ${ }^{4}$ APA is often associated with a major vascular occlusion like CRVO or CRAO. CRVO cases are more likely than controls to have high anticardiolipin antibody levels with an odds ratio of $6.26 .^{5}$ The first patient presented with CRVO, which involved major vascular occlusion and illustrates a classical presentation of APS; thus, she was screened for APA, which turned out to be positive and was started on an anticoagulant with oral warfarin. Standard treatment for initial venous thrombotic event is early initiation with oral warfarin. ${ }^{6}$

Mild lupus retinopathy consists of cotton wool spots, retinal hemorrhages, and vascular tortuosity, where as severe lupus retinopathy consists of occlusion of retinal arterioles leading to retinal infarction, hence termed vaso-occlusive retinopathy or retinal vasculitis. Although the second patient only presented with features of vaso-occlusive lupus retinopathy, screening for APS was done in view of recent history of DVT, which turned out to be positive as well. Initial management of lupus retinopathy includes systemic immunosuppression (usually intravenous corticosteroids followed by oral steroid), which is then supplemented or replaced by other immunosuppressive agents. However, if APA is present, prompt use of anticoagulant is beneficial, as APA is linked to focal thrombosis. ${ }^{7}$ Therefore, screening for APS in the second patient prevented a delay in initiating the anticoagulant and worsening of her condition.

We have highlighted two cases of SLE whereby the first case with CRVO illustrates a classical presentation of APS and the second case with features of active vaso-occlusive lupus retinopathy. However, APS can be present in SLE regardless of the nature of retinopathy. Hence, screening for APS is useful, as prompt treatment with anticoagulants is important to prevent further vascular thrombosis and worsening visual prognosis. Although systemic steroids and antithrombotic agent were given in these cases, visual prognosis was not good as a result of macular ischemia, leading to permanent visual disability. Nevertheless, the most important issue now is to prevent a similar condition in the fellow eye, therefore antiinflammatory and antithrombotic need to be given for a longer duration.

\section{Disclosure}

The authors report no conflicts of interest in this work.

\section{References}

1. Arevalo JF, Lowder CY, Muci-Mendoza R. Ocular manifestations of systemic lupus erythematosus. Curr Opin Ophthalmol. 2002;13(6): 404-410.

2. Sivaraj RR, Durrani OM, Denniston AK, Murray PI, Gordon C. Ocular manifestations of systemic lupus erythematosus. Rheumatology. 2007; 46(12):1757-1762.

3. Asherson RA, Mony P, Acheson JF, Harris EN, Hughes GR. Antiphospholipid syndrome: a risk factor for occlusive ocular vascular disease in systemic lupus erythematosus and the primary antiphospholipid syndrome. Ann Rheum Dis. 1989;48(5):358-361.

4. Durrani OM, Gordon C, Murray PT. Primary anti-phospholipid antibody syndrome (APS): current concept. Surv Ophthalmol. 2002;47(3): 215-238.

5. Glueck CJ, Hutchins RK, Jurantee J, Khan Z, Wang P. Thrombophilia and retinal vascular occlusion. Clin Ophthalmol. 2012;6:1377-1384.

6. Utz VM, Tang J. Ocular manifestations of the antiphospholipid syndrome. Br J Ophthalmol. 2011;95:454-459.

7. Au A, O'Day J. Review of severe vaso-occlusive retinopathy in systemic lupus erythematosus and the antiphospholipid syndrome: associations, visual outcomes, complications and treatment. Clin Experiment Ophthalmol. 2004;32(1):87-100. 
Clinical Ophthalmology

\section{Publish your work in this journal}

Clinical Ophthalmology is an international, peer-reviewed journal covering all subspecialties within ophthalmology. Key topics include: Optometry; Visual science; Pharmacology and drug therapy in eye diseases; Basic Sciences; Primary and Secondary eye care; Patien Safety and Quality of Care Improvements. This journal is indexed on

PubMed Central and CAS, and is the official journal of The Society of Clinical Ophthalmology (SCO). The manuscript management system is completely online and includes a very quick and fair peer-review system, which is all easy to use. Visit http://www.dovepress.com/ testimonials.php to read real quotes from published authors. 